\title{
OPEN Chemical profile and phytotoxic action of Onopordum acanthium essential oil
}

\author{
Caixia Wei ${ }^{1}$, Shixing Zhou ${ }^{2}$, Kai Shi ${ }^{3}$, Chi Zhang $^{4}$ \& Hua Shao ${ }^{1,2} \bowtie$
}

The potential of utilizing Onopordum acanthium essential oil and its major constituents as environment friendly herbicides was investigated. In total 29, 25, and 18 compounds were identified from flower, leaf, and stem oils, representing $94.77 \%, 80.02 \%$, and $90.74 \%$ of the total oil, respectively. Flower and stem oils were found to be rich in $n$-alkanes, which accounted for $\mathbf{5 7 . 3 3 \%}$ in flower oil, and $\mathbf{8 2 . 3 3 \%}$ in stem oil. Flower oil exerted potent inhibitory activity on both receiver species, Amaranthus retroflexus and Poa annua, which nearly completely suppressed seed germination at $5 \mathrm{mg} / \mathrm{mL}$, and $\beta$-eudesmol is the most likely responsible compound for its phytotoxicity; in comparison, leaf and stem oils exhibited much weaker inhibitory activity on $A$. retroflexus, and stimulatory effect on $P$. annua when tested concentration was below $2.5 \mathrm{mg} / \mathrm{mL}$. Alkanes in the oils were found to exert relatively weak plant growth regulatory activity. This report is the first on the chemical profile and phytotoxic action of $O$. acanthium oil as well as the phytotoxicity of $\beta$-eudesmol.

The genus Onopordum (Asteraceae) comprises 50 species that distribute in continental Europe and central and southwest of Asia ${ }^{1}$. Among them, O. acanthium L., also known as Scotch thistle, is a biennial species, and height of mature plants can reach up to $3 \mathrm{~m}$. Flowering plants have white lanate stems that are ridged with spinymargined wings; the plant bears purple flowers, the inflorescence is a panicle that bears capitula ${ }^{2}$. This plant is a competitive species against forage plants, and its flowering stalks form a barrier to livestock movement, which makes this plant an agricultural pest in the U.S., Canada, and other countries ${ }^{2}$. In China, O. acanthium can be found frequently as a dominant species distributing in the Tianshan mountains and the Junggar basin of Xinjiang province, which is known as the arid or semi-arid region located in northwest China ${ }^{3-5}$.

Worldwide, O. acanthium has been extensively used to treat cancers, inflammatory disorders, cardiovascular, and urogenital diseases ${ }^{6-12,13}$ For instance, Cardiodoron, a medicinal product made by O. acanthium, is well-tolerated for treatment of functional cardiovascular that shows positive effects in medical practice ${ }^{11}$. Phytochemical studies revealed that it might produce allelochemicals with phytotoxic activities, such as flavonoids and sesquiterpene lactones, which could attribute to its widespread over several continents ${ }^{14}$. Flower of O. acanthium is widely used as a coagulating agent in cheesemaking industry, and its young shoots as well as its first year roots are used in salads ${ }^{15,16}$. The oil obtained from $O$. acanthium can be used as a renewable fuel and chemical feedstock, and there are quite a few reports on the yield and chemical composition such as fatty acid composition, tocopherol, and mineral contents of its oil ${ }^{17-20}$.

Essential oils are a group of special natural substances known for biological activities such as antimicrobial, herbicidal, antioxidant, anti-inflammatory activities, and so on ${ }^{21-25}$. So far there is no report on the chemical constituents of $O$. acanthium essential oil. The objectives of the current study include: (i) determination of the chemical profile of the essential oils obtained from different plant organs of O. acanthium; (ii) evaluation of the phytotoxic activity of $O$. acanthium essential oils and the major components.

\footnotetext{
${ }^{1}$ State Key Laboratory of Desert and Oasis Ecology, Xinjiang Institute of Ecology and Geography, Chinese Academy of Sciences, Urumqi 830011, Xinjiang, China. ${ }^{2}$ Research Center for Ecology and Environment of Central Asia, Xinjiang Institute of Ecology and Geography, Chinese Academy of Sciences, Urumqi 830011, China. ${ }^{3}$ University of Chinese Academy of Sciences, Beijing 100049, China. ${ }^{4}$ Shandong Provincial Key Laboratory of Water and Soil Conservation and Environmental Protection, College of Resources and Environment, Linyi University, Linyi 276000, China. ${ }^{\square}$ email: shaohua@ms.xjb.ac.cn
} 


\section{Results}

Chemical constituents of 0 . acanthium essential oils extracted from different plant organs. Essential oils were extracted from fresh flowers, leaves, and stems of O. acanthium using hydrodistillation method. The phytochemical profiles of the essential oils obtained from different plant organs were analyzed via GC/MS, and the results were presented in Table 1 . In total, 29, 25, and 18 compounds were identified from flower, leaf, and stem oils, representing $94.77 \%, 80.02 \%$, and $90.74 \%$ of the total oil mass, respectively. All these oils were found to be rich in a type of particular compounds- $n$-alkanes (mainly C22-C29), such as tricosane, tetracosane, pentacosane, hexacosane, heptacosane, octacosane, nonacosane, etc., especially in flowers and stems, which accounted for $57.33 \%$ in the flower oil, and $82.33 \%$ in the stem oil. The major components of flower, leaf and stem oils differed significantly: pentacosane $(12.41 \%)$, hexacosane (10.44\%), $\beta$-eudesmol $(10.10 \%)$, heptacosane $(8.67 \%)$, tetracosane $(8.43 \%)$, and tricosane $(5.36 \%)$ were dominant in flower oil; leaf oil contain 1-Hexanol (14.88\%), 3-Hexen-1-ol, (E)- (13.53\%), 2-Hexen-1-ol, (E)- (12.18\%), 2-Hexenal, (E)(6.93\%), nonanal (9.74\%); on the other hand, stem oil was rich in nonacosane (16.44\%), nonacosane, 2-methyl(15.54\%), hentriacontane (15.05\%), and dotriacontane $(13.73 \%)$.

Phytotoxic activity of flower, leaf and stem oils of $O$. acanthium. Flower, leaf, and stem oils (concentration tested ranged from $0.25-5 \mathrm{mg} / \mathrm{mL}$ ) all exhibited significant plant growth regulatory activity against both receiver species (Figs. 1,5). The oils posed inhibitory activity on root development of the dicot plant, A. retroflexus, with flower oil being the most potent oil, suppressing root elongation by $12.92 \%$ and $14.25 \%$ at 0.25 and $0.5 \mathrm{mg} / \mathrm{mL}$. When the concentration of flower oil was increased to $1 \mathrm{mg} / \mathrm{mL}$, the inhibitory effect was enhanced markedly, reaching $54.3 \%$ compared with the control. The suppressive effect was enhanced to $75.49 \%$ by $2.5 \mathrm{mg} /$ $\mathrm{mL}$ oil, meanwhile $5 \mathrm{mg} / \mathrm{mL}$ flower oil almost completely killed the seedlings (99.60\%).

Although leaf and stem oils also exhibited negative impact on root growth of $A$. retroflexus, the strength was much weaker. Root length decreased to $6.89-33.66 \%$ of control when treated with leaf oil ranging from $0.25-5 \mathrm{mg} / \mathrm{mL}$, and $9.15-26.28 \%$ with stem oil. It was obvious that strength of the oils' phytotoxicity was much weaker compared with the commercial herbicide glyphosate, which reduced root length by $71.22 \%, 78.75 \%$, $91.94 \%, 95.66 \%$ and $96.20 \%$ at $0.25,0.5,1,2$, and $5 \mathrm{mg} / \mathrm{mL}$, respectively. Shoot development of $A$. retroflexus responded to the oils in a similar way, however both leaf and stem oils promoted shoot length by $3.91 \%$ and $5.91 \%$, respectively (Figs. 2,5 ).

Flower oil exhibited similar inhibitory activity on $P$. annua, which was also much more sensitive to flower oil than leaf and stem oils. Root length was reduced to $49.75 \%$ and $59.72 \%$ of the control when treated with flower oil at $1 \mathrm{mg} / \mathrm{mL}$ and $2.5 \mathrm{mg} / \mathrm{mL}$, respectively, and $5 \mathrm{mg} / \mathrm{mL}$ almost completely prohibited seed germination of $P$. аппиа (Figs. 3, 5). Similarly, glyphosate presented much stronger phytotoxic effect, inhibiting root elongation by $79.08 \%$ and $93.44 \%$ at 0.25 and $0.5 \mathrm{mg} / \mathrm{mL}$, respectively, and starting from $1 \mathrm{mg} / \mathrm{mL}$, seedling development was completely prohibited. On the other hand, leaf and stem oils promoted root development except the highest concentration tested $(5 \mathrm{mg} / \mathrm{mL})$, which reduced root length by $12.05 \%$ and $0.98 \%$; leaf and stem oils stimulated root elongation by $8.13-22.11 \%$ and $4.14-14.41 \%$, respectively. Like $A$. retroflexus, shoot growth of P. annua also displayed the same pattern as root growth (Figs. 4, 5).

Phytotoxic activity of alkanes as major constituents of the essential oils. The essential oils produced by $O$. acanthium were rich in various alkane compounds, especially the flower and stem oils. Therefore, their possible involvement of the plant regulatory activity was assessed. In general, alkanes as the major constituents of the essential oils possessed relatively weak plant growth regulatory activity on receiver species. In terms of A. retroflex, alkanes stimulated root elongation by up to $26.97 \%$ (tetracosane at $0.25 \mathrm{mg} / \mathrm{mL}$ ) when the applied concentration was between $0.25-0.5 \mathrm{mg} / \mathrm{mL}$, and the strongest inhibitory effect $(13.12 \%)$ was observed when the mixture of different alkanes were mixed at $5 \mathrm{mg} / \mathrm{mL}$ (Table 2). For A. retroflex shoot, only suppressive effect occurred, with pentacosane inhibiting shoot growth by $37.42 \%$ at $2.5 \mathrm{mg} / \mathrm{mL}$ (Table 3). As of P. annua, in general root development was inhibited, and the highest value was triggered by application of nonacosane at $5 \mathrm{mg} / \mathrm{mL}$ (Table 4); meanwhile, shoot length was promoted when alkane concentrations were lower than $2.5 \mathrm{mg} / \mathrm{mL}$, and tricosane at $0.25 \mathrm{mg} / \mathrm{mL}$ enhanced shoot growth by $20.12 \%$ compared to the control. The mixture exhibited the most potent suppressive effect, resulting in $17.59 \%$ reduction on shoot elongation of $P$. annua (Table 5).

Phytotoxic activity of $\beta$-eudesmol. Phytotoxic assay demonstrated that the strength of flower oil was much higher than leaf and stem oils; further comparison revealed that flower oil was abundant in a unique compound, $\beta$-eudesmol. It is thus speculated that this compound might be responsible for the phytotoxic activity of the flower oil. To testify this hypothesis, the phytotoxic activity of $\beta$-eudesmol was further evaluated, and the results showed that indeed it possessed the same biological activity comparable to the flower oil (Tables 6 , 7; Fig. 5). Starting from the lowest concentration, $0.25 \mathrm{mg} / \mathrm{mL}$, over $50 \%$ reduction of root and shoot growth of both receiver plants were observed: root length was reduced by $79.46 \%$ and $63.34 \%$ for $A$. retroflexus and $P$. annua, and shoot length was suppressed by $60.37 \%$ and $68.96 \%$, respectively. When the concentration was doubled to $0.5 \mathrm{mg} / \mathrm{mL}$, seedling growth was nearly completely inhibited, and further increase in the dose resulted in $100 \%$ death of the seeds. The content of $\beta$-eudesmol was $10.16 \%$ in the total oil, therefore, if $\beta$-eudesmol was the only constituent responsible for the phytotoxicity of the oil, the phytotoxicity of $0.25 \mathrm{mg} / \mathrm{mL} \beta$-eudesmol should be similar to $2.5 \mathrm{mg} / \mathrm{mL}$ oil, whilest $0.5 \mathrm{mg} / \mathrm{mL} \beta$-eudesmol equals to the activity triggered by $5 \mathrm{mg} / \mathrm{mL}$ oil. In the assay, $0.25 \mathrm{mg} / \mathrm{mL} \beta$-eudesmol caused $79.46 \%$ and $63.34 \%$ root reduction of $A$. retroflexus and $P$. annua, compared with $75.49 \%$ and $59.72 \%$ affected by flower oil at $2.5 \mathrm{mg} / \mathrm{mL}$. Meanwhile, $0.5 \mathrm{mg} / \mathrm{mL} \beta$-eudesmol caused $98.81 \%$ and $100 \%$ inhibition rate on root elongation of A. retroflexus and P. annua, compared with $99.6 \%$ and $100 \%$ on test species. In terms of shoot development, $0.25 \mathrm{mg} / \mathrm{mL} \beta$-eudesmol caused $60.37 \%$ and $68.96 \%$ reduc- 


\begin{tabular}{|c|c|c|c|c|}
\hline Compounds & Number & Flower (\%) & Leaf $(\%)$ & Stem (\%) \\
\hline 2-Hexenal, (E)- & 1 & - & 6.93 & - \\
\hline 3-Hexen-1-ol, (E)- & 2 & - & 13.53 & 1.17 \\
\hline 2-Hexen-1-ol, (E)- & 3 & - & 12.18 & - \\
\hline 1-Hexanol & 4 & - & 14.88 & - \\
\hline Heptanal & 5 & - & 0.68 & - \\
\hline 2-Amylfuran & 6 & - & 0.73 & - \\
\hline (3E)-Hexenyl acetate & 7 & - & 1.19 & - \\
\hline Nonanal & 8 & - & 9.74 & 1.01 \\
\hline$(+)$-2-Bornanone & 9 & - & 0.11 & - \\
\hline Methyl salicylate & 10 & - & 0.16 & - \\
\hline 3-p-Menthen-7-al & 11 & - & 0.48 & - \\
\hline 2-Decenal, (Z)- & 12 & - & 0.33 & - \\
\hline Undecanal & 13 & 0.28 & 0.71 & - \\
\hline Tridecane & 14 & - & 1.00 & 0.10 \\
\hline 2-Buten-1-one, 1-(2,6,6-trimethy... & 15 & - & - & 0.16 \\
\hline$\beta$-Damascenone & 16 & 0.89 & 1.70 & - \\
\hline$\beta$-Ionone & 17 & - & 1.01 & - \\
\hline Phenol, 2,4-di-tert-butyl- & 18 & 0.65 & - & - \\
\hline Pentadecane & 19 & - & - & 0.73 \\
\hline Farnesan & 20 & - & 0.61 & - \\
\hline Elemol & 21 & 1.94 & - & - \\
\hline D-Nerolidol & 22 & 1.53 & - & - \\
\hline Farnesene epoxide, E- & 23 & 1.77 & - & - \\
\hline 7-epi- $\gamma$-Eudesmol & 24 & 1.17 & - & - \\
\hline$\beta$-Eudesmol & 25 & 10.10 & - & - \\
\hline Bisabolol & 26 & 0.65 & - & - \\
\hline Hexadecane & 27 & - & - & 0.25 \\
\hline Laciniatafuranone $\mathrm{F}$ & 28 & - & 0.36 & - \\
\hline cis-Davanone & 29 & - & 5.91 & - \\
\hline 1,2-Epoxyhexadecane & 30 & 3.20 & - & - \\
\hline Heptadecane & 31 & - & - & 4.71 \\
\hline Z-11-Hexadecenoic acid & 32 & 1.38 & - & - \\
\hline 9,12-Octadecadienal & 33 & 2.30 & - & - \\
\hline Hexahydrofarnesyl acetone & 34 & 2.17 & - & - \\
\hline Nonadecane & 35 & - & - & 2.19 \\
\hline Dodecenyl succinic anhydride & 36 & 0.09 & - & - \\
\hline Hexahydrofarnesyl acetone & 37 & 1.59 & - & - \\
\hline 2-Nonadecanone & 38 & 0.12 & - & - \\
\hline Dibutyl phthalate & 39 & 3.26 & - & 3.26 \\
\hline Phytol & 40 & 2.06 & - & - \\
\hline Docosane & 41 & 1.35 & - & - \\
\hline Tricosane & 42 & 5.36 & - & - \\
\hline Octadecyl bromide & 43 & - & 0.17 & - \\
\hline Phthalic acid & 44 & - & 2.00 & - \\
\hline Tetracosane & 45 & 8.43 & - & 3.31 \\
\hline Pentacosane & 46 & 12.41 & - & - \\
\hline Hexacosane & 47 & 10.44 & 0.44 & 0.09 \\
\hline Heptacosane & 48 & 8.67 & - & - \\
\hline Octacosane & 49 & 0.79 & - & 17.32 \\
\hline 7-Hexyldocosane & 50 & 4.45 & - & - \\
\hline 1-Nonacosene & 51 & - & 0.13 & - \\
\hline 2-Methyloctacosane & 52 & - & 4.34 & 0.22 \\
\hline Nonacosane & 53 & 3.37 & - & 16.44 \\
\hline Triacontane & 54 & - & - & 0.53 \\
\hline Nonacosane, 2-methyl- & 55 & - & - & 15.54 \\
\hline Hentriacontane & 56 & 2.06 & - & 15.05 \\
\hline
\end{tabular}




\begin{tabular}{|l|l|l|l|l|}
\hline Compounds & Number & Flower (\%) & Leaf (\%) & Stem (\%) \\
\hline Dotriacontane & 57 & - & - & 13.73 \\
\hline Tetradecamethylhexasiloxane & 58 & - & 0.70 & - \\
\hline Total & & 94.77 & 80.02 & 90.74 \\
\hline
\end{tabular}

Table 1. Chemical composition of the essential oils extracted from flowers, leaves and stems of O. acanthium.

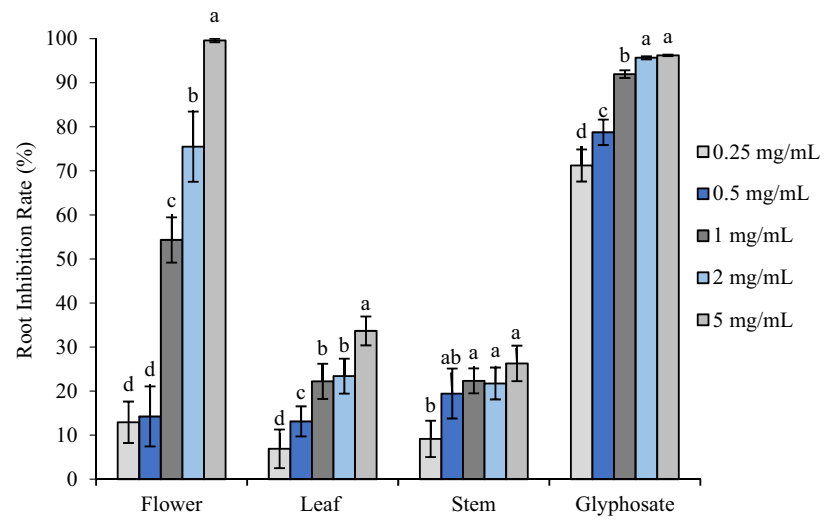

Figure 1. Phytotoxic effect of flower, leaf and stem oil of $O$. acanthium as well as glyphosate on radical elongation of $A$. retroflexus $(n=30)$. Each value is the mean of three replicates $\pm \mathrm{SE}(n=30)$. Significant difference $(p<0.05)$ was represented by different letters.

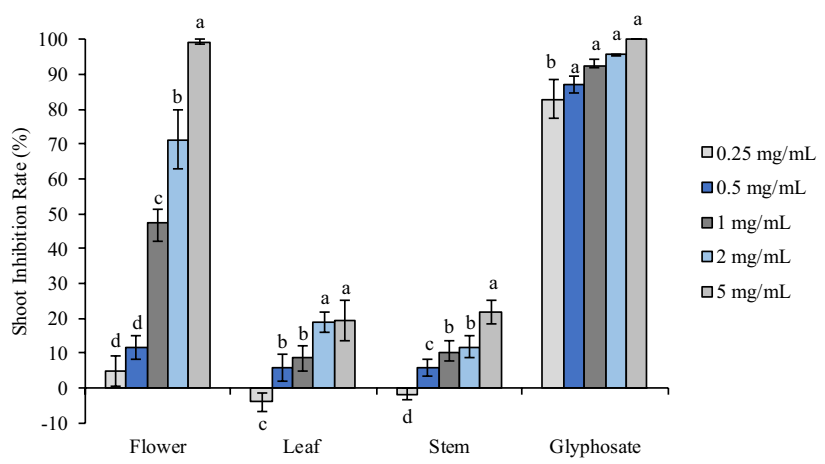

Figure 2. Phytotoxic effect of flower, leaf and stem oil of $O$. acanthium as well as glyphosate on shoot elongation of $A$. retroflexus. Each value is the mean of three replicates \pm SE $(n=30)$. Significant difference $(p<0.05)$ was represented by different letters.

tion of A. retroflexus and P. annua, compared with $71.31 \%$ and $60.20 \%$ of reduction under $2.5 \mathrm{mg} / \mathrm{mL}$ flower oil treatment; meanwhile, $0.5 \mathrm{mg} / \mathrm{mL} \beta$-eudesmol reduced shoot elongation by $97.41 \%$ and $100 \%$, compared with $99.43 \%$ and $98.76 \%$ triggered by $5 \mathrm{mg} / \mathrm{mL}$ flower oil. Thus, it was concluded that the single compound $\beta$-eudesmol was most likely the active responsible compound for the strong phytotoxicity of flower oil.

\section{Discussion}

Our study is the only report so far on the chemical composition and phytotoxicity of O. acanthium essential oil. Previously, the chemical composition of essential oils from another Onopordum species, O. arenarium, was investigated, and in total 29 and 25 compounds were identified from this plant's flower and stem volatile oils, representing $91.6 \%$ and $89.2 \%$ of the oil mass, respectively. The oils were found to possess antioxidant activity, which were rich in long-chain hydrocarbons (23.3-36.4\%), oxygenated long-chain hydrocarbons (31.5-33.8\%), and oxygenated monoterpenes (14.4-6.6\%), with palmitic acid being the most abundant constituent $(25.5-28.7 \%)^{26}$. As of non-volatile compounds, phytochemical study revealed the presence of flavonoids such as apigenin, luteolin, scutellarein, coumarins such as aesculin and aesculetin, sesquiterpene lactones including derivatives of elemane, germacrane, eudesmane, and guaiane ${ }^{1}$. Watanabe et al. (2014) suspected that allelopathy might facilitate the invasion success of $O$. acanthium, therefore bioassay-guided isolation procedure was applied using wheat seeds as test species to investigate the chemicals in the leaves, which was extracted with different solvents; the $\mathrm{CH}_{2} \mathrm{Cl}_{2}$ 


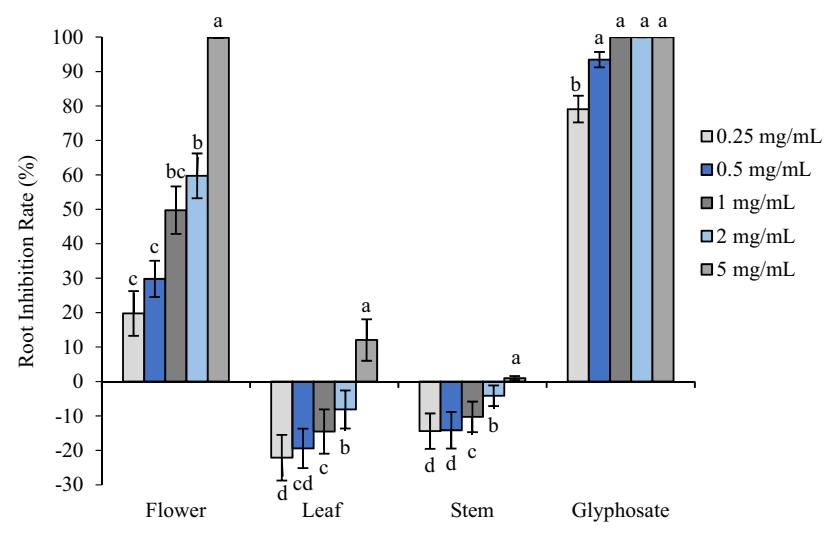

Figure 3. Phytotoxic effect of flower, leaf and stem oil of $O$. acanthium as well as glyphosate on radical elongation of $P$. annua. Each value is the mean of three replicates \pm SE $(n=30)$. Significant difference $(p<0.05)$ was represented by different letters.

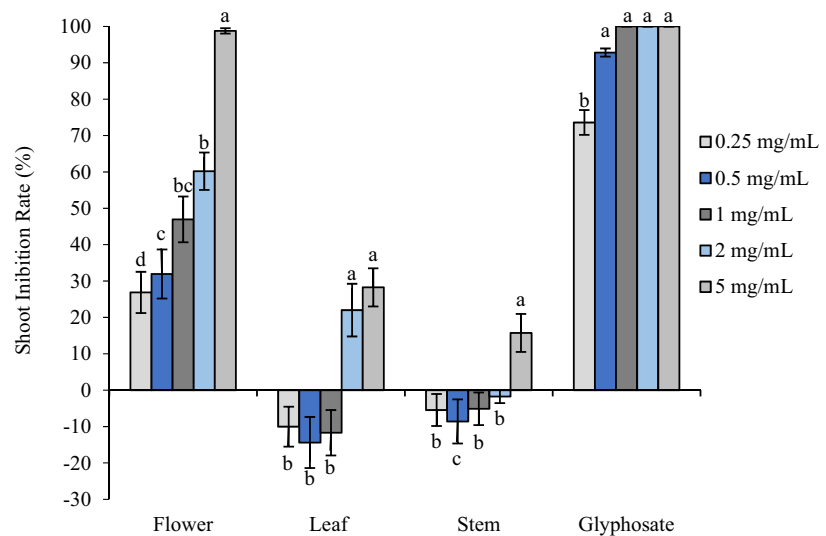

Figure 4. Phytotoxic effect of flower, leaf and stem oil of $O$. acanthium as well as glyphosate on shoot elongation of $P$. аnnua. Each value is the mean of three replicates \pm SE $(n=30)$. Significant difference $(p<0.05)$ was represented by different letters.

extract showed the most potent phytotoxic activity, which was then further purified to yield 4 compounds, i.e. two flavonoids, pectolarigenin (1) and scutellarein 4'-methyl ether (2), and two sesquiterpene lactones, elemanolide 11(13)-dehydromelitensin b-hydroxyisobutyrate (3) and acanthiolide (4), among which they found compound 3 $\left(\mathrm{IC}_{50}=1.794 \times 10^{-4} \mathrm{M}\right)$ strongly inhibited the growth of wheat coleoptiles and compound $1\left(\mathrm{IC}_{50}=1.263 \times 10^{-3} \mathrm{M}\right)$ showed an intermediate effect ${ }^{14}$. To the best of knowledge, this is the first study on the chemical profile and phytotoxicity of this plant's essential oil. Altogether 60 compounds from flower, leaf and stem oils of O. acanthium were identified, which were found to be rich in higher $n$-alkanes.

Alkanes are important industrial chemicals and energy resources that can be found rich in petroleum and natural gas. Although a number of plant species are found to produce essential oils containing alkanes as constituents, the amount is usually relatively low, except a single report on the chemical profile of Asphodelus aestivus flower essential oil, which was abundant in hexadecanoic acid (35.6\%), pentacosane (17.4\%), tricosane (13.4\%), heptacosane $(8.4 \%)$, heneicosane $(4.5 \%)$, tetracosane (3.0\%), and hexacosane (2.0). Polatoğlu et al. also identified nonacosane $(16.18 \pm 0.13 \%)$ and heptacosane $(14.91 \pm 0.17 \%)$ from the flower oil of Arabis purpurea Poech oil; it is interesting that similar to the current study, this oil was also obtained from the flowers of the donor plant ${ }^{27}$.

Besides, alkanes have been reported to be present in essential oils extracted from other Asteraceae plants. For instance, alkanes (C11-C27) accounted for $4.12 \%$ of the total mass of the essential oil of Centaurea raphanina, and $6.1 \%$ of C. spruneri ${ }^{28}$; other reports include that Hemizonia fitchii oil contained $1.7 \%$ alkanes (C25-C29) ${ }^{29}$, and $0.9 \%$ in Sigesbeckia jorullensis oil ${ }^{30}$. Compared with published studies, alkanes (C22-C29) including tricosane, tetracosane, pentacosane, hexacosane, heptacosane, octacosane, nonacosane accounted for $57.33 \%$ in flower oil, and $82.33 \%$ in stem oil, indicating the potential value of utilizing $O$. acanthium as a natural source to produce alkanes for industrial uses. It is noteworthy to mention that $O$. acanthium is a widespread species with high biomass that can adapt to various environmental conditions including arid and barren habitats, which makes it practical to be cultivated in large scale for industrial purpose.

This study indicated that flower oil possessed much stronger inhibitory effect compared with leaf and stem oils: the inhibition rate of flower oil on root growth of $A$. retroflexus was 2.96 and 3.79 times of leaf and stem oils, 

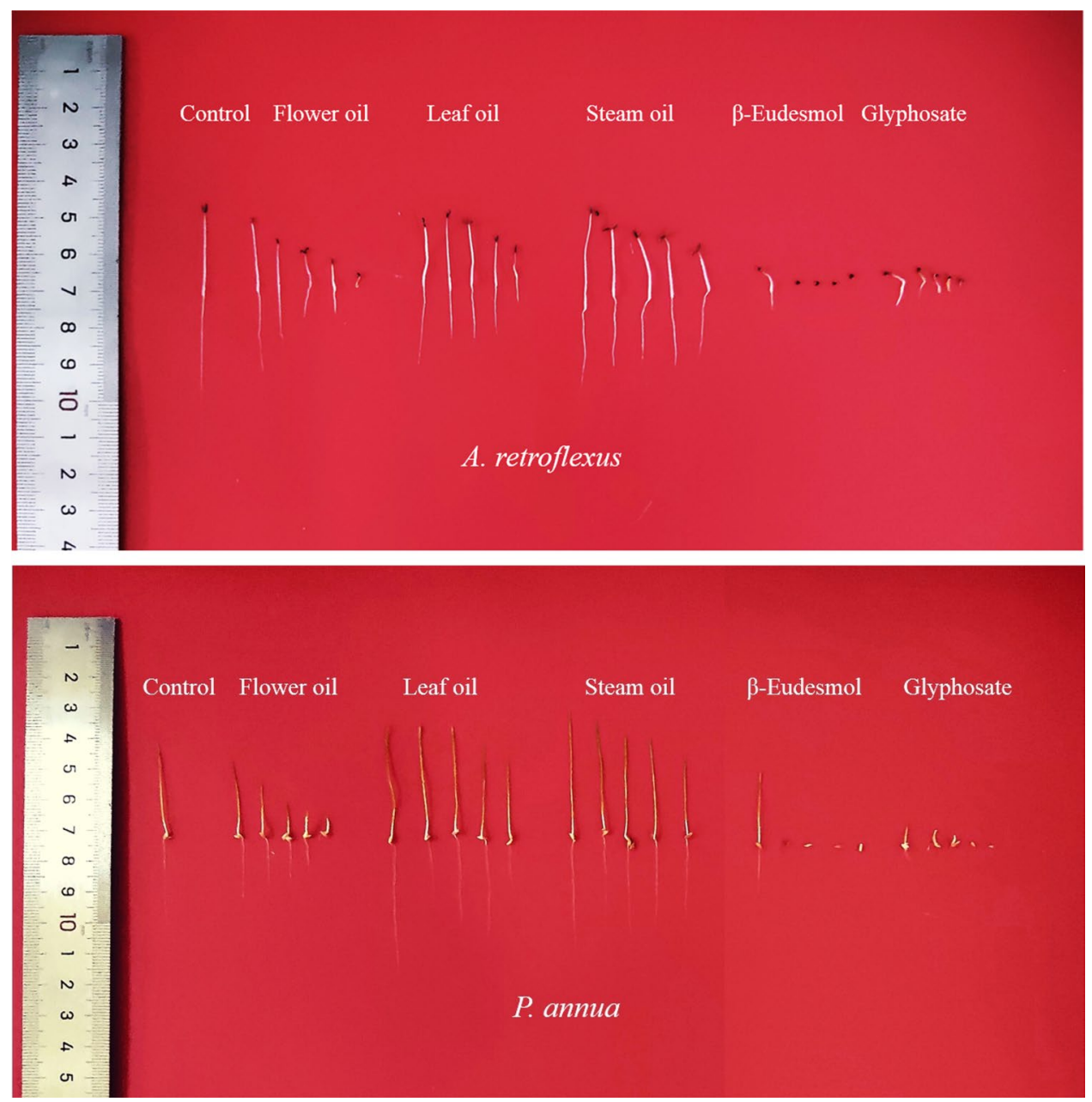

Figure 5. Phytotoxic effect of flower, leaf, and stem oil of O. acanthium, $\beta$-eudesmol, and glyphosate (concentration tested ranging from $0.25-5 \mathrm{mg} / \mathrm{mL}$ ) on seedling growth of $A$. retroflexus and $P$. annua.

\begin{tabular}{|c|c|c|c|c|c|c|c|c|c|}
\hline & $\begin{array}{l}\text { Concentration } \\
(\mathrm{mg} / \mathrm{mL})\end{array}$ & Tricosane & Tetracosane & Pentacosane & Hexacosane & Heptacosane & Octacosane & Nonacosane & Mixture \\
\hline \multirow{5}{*}{$\begin{array}{l}\text { A. retroflexus } \\
\text { Root inhibi- } \\
\text { tion }(\%)\end{array}$} & 0.25 & $-10.22 \pm 2.31^{b}$ & $-26.97 \pm 3.33^{b}$ & $-9.99 \pm 1.82^{\mathrm{a}}$ & $-16.38 \pm 4.21^{\mathrm{c}}$ & $-12.10 \pm 4.41^{b}$ & $-9.29 \pm 3.18^{\mathrm{a}}$ & $-14.09 \pm 3.20^{b}$ & $-15.26 \pm 5.00^{c}$ \\
\hline & 0.5 & $-10.46 \pm 4.41^{b}$ & $-17.78 \pm 4.13^{\mathrm{ab}}$ & $-4.01 \pm 1.10^{\mathrm{a}}$ & $-13.70 \pm 4.0^{\mathrm{bc}}$ & $-9.05 \pm 2.30^{\mathrm{b}}$ & $-4.81 \pm 1.24^{\mathrm{a}}$ & $-14.26 \pm 3.23^{b}$ & $-16.67 \pm 9.33^{c}$ \\
\hline & 1 & $-2.38 \pm 1.10^{\mathrm{ab}}$ & $-19.71 \pm 4.70^{\mathrm{ab}}$ & $0.76 \pm 0.47^{\mathrm{a}}$ & $-9.78 \pm 4.89^{b}$ & $-1.91 \pm 1.10^{\mathrm{ab}}$ & $-2.73 \pm 1.55^{\mathrm{a}}$ & $-3.19 \pm 3.92^{\mathrm{a}}$ & $2.17 \pm 4.29^{\mathrm{b}}$ \\
\hline & 2.5 & $-3.31 \pm 3.10^{\mathrm{ab}}$ & $-18.31 \pm 3.65^{a b}$ & $0.90 \pm 0.49^{\mathrm{a}}$ & $-1.32 \pm 1.92^{\mathrm{a}}$ & $-2.38 \pm 2.31^{\mathrm{ab}}$ & $-0.38 \pm 0.34^{\mathrm{a}}$ & $-6.03 \pm 3.25^{\mathrm{a}}$ & $1.96 \pm 1.93^{\mathrm{b}}$ \\
\hline & 5 & $8.05 \pm 3.43^{\mathrm{a}}$ & $-9.06 \pm 3.81^{\mathrm{a}}$ & $2.29 \pm 0.18^{\mathrm{a}}$ & $-0.38 \pm 0.28^{\mathrm{a}}$ & $7.67 \pm 3.43^{\mathrm{a}}$ & $-0.77 \pm 0.91^{\mathrm{a}}$ & $1.26 \pm 2.69^{\mathrm{a}}$ & $13.12 \pm 3.55^{\mathrm{a}}$ \\
\hline
\end{tabular}

Table 2. Phytotoxic activity of alkanes on root growth of A. retroflexus. ${ }^{\mathrm{a}} \pm$ Standard error $(n=30) .{ }^{\mathrm{b}}$ Different letters represent a significant difference at $p<0.05$ level according to Fisher's LSD test.

\begin{tabular}{|c|c|c|c|c|c|c|c|c|c|}
\hline & $\begin{array}{l}\text { Concentration } \\
(\mathrm{mg} / \mathrm{mL})\end{array}$ & Tricosane & Tetracosane & Pentacosane & Hexacosane & Heptacosane & Octacosane & Nonacosane & Mixture \\
\hline \multirow{5}{*}{$\begin{array}{l}\text { A. } \\
\text { retro- } \\
\text { flexus } \\
\text { Shoot } \\
\text { inhi- } \\
\text { bition } \\
(\%)\end{array}$} & 0.25 & $0.08 \pm 0.55^{\mathrm{b}}$ & $14.28 \pm 2.62^{b}$ & $22.06 \pm 3.49^{b}$ & $24.31 \pm 1.93^{\mathrm{a}}$ & $20.30 \pm 4.06^{\mathrm{b}}$ & $11.28 \pm 2.85^{\mathrm{b}}$ & $20.45 \pm 2.97^{\mathrm{a}}$ & $14.96 \pm 2.79^{\mathrm{a}}$ \\
\hline & 0.5 & $0.07 \pm 0.80^{\mathrm{a}}$ & $14.79 \pm 3.04^{\mathrm{b}}$ & $24.55 \pm 3.39^{b}$ & $23.06 \pm 3.25^{\mathrm{a}}$ & $28.21 \pm 2.49^{\mathrm{a}}$ & $17.54 \pm 2.80^{\mathrm{a}}$ & $19.55 \pm 3.77^{\mathrm{a}}$ & $17.29 \pm 2.97^{\mathrm{a}}$ \\
\hline & 1 & $0.10 \pm 0.40^{\mathrm{a}}$ & $13.87 \pm 1.92^{\mathrm{b}}$ & $29.07 \pm 2.41^{\mathrm{ab}}$ & $25.78 \pm 0.08^{\mathrm{a}}$ & $28.32 \pm 3.40^{\mathrm{a}}$ & $19.30 \pm 2.52^{\mathrm{a}}$ & $22.81 \pm 4.41^{\mathrm{a}}$ & $16.79 \pm 2.47^{\mathrm{a}}$ \\
\hline & 2.5 & $0.16 \pm 0.89^{\mathrm{a}}$ & $16.04 \pm 1.91^{b}$ & $37.42 \pm 4.54^{\mathrm{a}}$ & $27.57 \pm 13.77^{\mathrm{a}}$ & $31.58 \pm 3.27^{\mathrm{a}}$ & $21.30 \pm 2.46^{\mathrm{a}}$ & $23.56 \pm 4.96^{\mathrm{a}}$ & $19.55 \pm 3.10^{\mathrm{a}}$ \\
\hline & 5 & $0.14 \pm 0.10^{\mathrm{a}}$ & $30.00 \pm 4.16^{\mathrm{a}}$ & $36.84 \pm 3.47^{\mathrm{a}}$ & $29.74 \pm 9.89^{\mathrm{a}}$ & $30.52 \pm 4.01^{\mathrm{a}}$ & $21.70 \pm 2.73^{\mathrm{a}}$ & $23.78 \pm 4.12^{\mathrm{a}}$ & $19.23 \pm 3.00^{\mathrm{a}}$ \\
\hline
\end{tabular}

Table 3. Phytotoxic activity of alkanes on shoot growth of A. retroflexus. ${ }^{\mathrm{a}} \pm$ Standard error $(n=30)$. ${ }^{\mathrm{b}}$ Different letters represent a significant difference at $p<0.05$ level according to Fisher's LSD test. 


\begin{tabular}{|c|c|c|c|c|c|c|c|c|c|}
\hline & $\begin{array}{l}\text { Concentration } \\
(\mathrm{mg} / \mathrm{mL})\end{array}$ & Tricosane & Tetracosane & Pentacosane & Hexacosane & Heptacosane & Octacosane & Nonacosane & Mixture \\
\hline \multirow{5}{*}{$\begin{array}{l}P . \\
\text { annua } \\
\text { Root } \\
\text { inhibi- } \\
\text { tion } \\
(\%)\end{array}$} & 0.25 & $7.42 \pm 3.79^{b}$ & $0.53 \pm 0.47^{b}$ & $-8.13 \pm 2.64^{b}$ & $-0.68 \pm 0.89^{b}$ & $16.54 \pm 2.66^{\mathrm{a}}$ & $0.06 \pm 0.03^{\mathrm{a}}$ & $8.66 \pm 3.20^{\mathrm{b}}$ & $7.29 \pm 3.77^{b}$ \\
\hline & 0.5 & $7.29 \pm 3.28^{b}$ & $-1.26 \pm 1.13^{\mathrm{b}}$ & $4.11 \pm 2.52^{\mathrm{a}}$ & $5.21 \pm 3.11^{\mathrm{b}}$ & $18.80 \pm 2.73^{\mathrm{a}}$ & $0.14 \pm 0.02^{\mathrm{a}}$ & $11.02 \pm 2.59^{\mathrm{ab}}$ & $11.83 \pm 3.51^{b}$ \\
\hline & 1 & $10.03 \pm 3.55^{\mathrm{ab}}$ & $4.41 \pm 3.23^{\mathrm{b}}$ & $3.39 \pm 2.38^{\mathrm{a}}$ & $14.40 \pm 2.79^{\mathrm{a}}$ & $20.30 \pm 2.81^{\mathrm{a}}$ & $0.13 \pm 0.30^{\mathrm{a}}$ & $12.79 \pm 2.43^{\mathrm{ab}}$ & $16.26 \pm 3.84^{\mathrm{b}}$ \\
\hline & 2.5 & $13.98 \pm 3.83^{\mathrm{a}}$ & $16.26 \pm 2.02^{\mathrm{a}}$ & $6.46 \pm 2.10^{\mathrm{a}}$ & $16.03 \pm 3.01^{\mathrm{a}}$ & $21.80 \pm 3.11^{\mathrm{a}}$ & $0.18 \pm 0.33^{\mathrm{a}}$ & $19.30 \pm 2.93^{\mathrm{ab}}$ & $20.90 \pm 2.75^{\mathrm{ab}}$ \\
\hline & 5 & $15.97 \pm 2.96^{\mathrm{a}}$ & $15.34 \pm 2.39^{\mathrm{a}}$ & $10.11 \pm 3.02^{\mathrm{a}}$ & $16.57 \pm 2.76^{\mathrm{a}}$ & $21.31 \pm 2.68^{\mathrm{a}}$ & $0.22 \pm 0.04^{\mathrm{a}}$ & $30.67 \pm 2.30^{\mathrm{a}}$ & $27.44 \pm 2.37^{\mathrm{a}}$ \\
\hline
\end{tabular}

Table 4. Phytotoxic activity of alkanes on root growth of $P$. annua. ${ }^{\mathrm{a}} \pm$ Standard error $(n=30) .{ }^{\mathrm{b}}$ Different letters represent a significant difference at $p<0.05$ level according to Fisher's LSD test.

\begin{tabular}{|c|c|c|c|c|c|c|c|c|c|}
\hline & $\begin{array}{l}\text { Concentration } \\
(\mathrm{mg} / \mathrm{mL})\end{array}$ & Tricosane & Tetracosane & Pentacosane & Hexacosane & Heptacosane & Octacosane & Nonacosane & Mixture \\
\hline \multirow{5}{*}{$\begin{array}{l}P . \\
\text { annua } \\
\text { Shoot } \\
\text { inhi- } \\
\text { bition } \\
(\%)\end{array}$} & 0.25 & $-20.12 \pm 3.25^{b}$ & $-6.23 \pm 2.61^{b}$ & $-16.93 \pm 3.04^{\mathrm{a}}$ & $2.99 \pm 4.32^{\mathrm{ab}}$ & $-8.82 \pm 2.46^{b}$ & $-16.58 \pm 3.43^{b}$ & $-12.63 \pm 3.31^{b}$ & $-2.02 \pm 1.36^{c}$ \\
\hline & 0.5 & $-19.21 \pm 2.95^{b}$ & $-4.21 \pm 2.65^{b}$ & $-13.16 \pm 2.61^{a}$ & $2.62 \pm 3.59^{\mathrm{a}}$ & $-9.68 \pm 3.33^{b}$ & $-12.22 \pm 4.85^{b}$ & $-10.26 \pm 2.80^{b}$ & $-9.47 \pm 4.19^{b}$ \\
\hline & 1 & $-12.63 \pm 2.88^{b}$ & $-4.65 \pm 4.11^{b}$ & $-12.43 \pm 2.82^{\mathrm{a}}$ & $2.94 \pm 3.84^{\mathrm{a}}$ & $-4.12 \pm 2.98^{\mathrm{ab}}$ & $-3.90 \pm 2.29^{\mathrm{ab}}$ & $-11.23 \pm 3.65^{b}$ & $-13.84 \pm 3.15^{b}$ \\
\hline & 2.5 & $-7.98 \pm 4.1^{\mathrm{ab}}$ & $-0.68 \pm 3.70^{\mathrm{ab}}$ & $-13.14 \pm 3.03^{\mathrm{a}}$ & $3.13 \pm 2.79^{b}$ & $-1.14 \pm 1.02^{\mathrm{a}}$ & $-4.47 \pm 4.90^{\mathrm{ab}}$ & $-5.18 \pm 3.23^{\mathrm{ab}}$ & $-11.85 \pm 2.25^{b}$ \\
\hline & 5 & $2.00 \pm 3.41^{\mathrm{a}}$ & $8.71 \pm 2.98^{\mathrm{a}}$ & $-7.13 \pm 4.77^{\mathrm{a}}$ & $2.36 \pm 3.24^{\mathrm{ab}}$ & $2.46 \pm 3.00^{\mathrm{a}}$ & $7.63 \pm 2.7^{\mathrm{a}}$ & $5.26 \pm 4.70^{\mathrm{a}}$ & $17.59 \pm 1.28^{\mathrm{a}}$ \\
\hline
\end{tabular}

Table 5. Phytotoxic activity of alkanes on shoot growth of $P$. annua. ${ }^{\mathrm{a}} \pm$ Standard error $(n=30) .{ }^{b}$ Different letters represent a significant difference at $p<0.05$ level according to Fisher's LSD test.

\begin{tabular}{|l|l|l|l|l|}
\hline \multirow{2}{*}{ Concentration $(\mathbf{m g} / \mathbf{m L})$} & \multicolumn{2}{l|}{ Root inhibition (\%) } & \multicolumn{2}{l|}{ Shoot inhibition (\%) } \\
\cline { 2 - 5 } & $\beta$-Eudesmol & Glyphosate & $\beta$-Eudesmol & Glyphosate \\
\hline 0.25 & $79.46 \pm 3.25^{\mathrm{b}}$ & $71.22 \pm 0.35^{\mathrm{d}}$ & $60.37 \pm 6.21^{\mathrm{b}}$ & $83.02 \pm 0.75^{\mathrm{e}}$ \\
\hline 0.5 & $98.81 \pm 0.68^{\mathrm{a}}$ & $78.75 \pm 0.46^{\mathrm{c}}$ & $97.41 \pm 1.57^{\mathrm{a}}$ & $87.05 \pm 0.25^{\mathrm{d}}$ \\
\hline 1 & $100 \pm 0.00^{\mathrm{a}}$ & $91.94 \pm 0.19^{\mathrm{b}}$ & $100 \pm 0.00^{\mathrm{a}}$ & $92.67 \pm 0.20 \mathrm{c}$ \\
\hline 2.5 & $100 \pm 0.00^{\mathrm{a}}$ & $95.66 \pm 0.13^{\mathrm{a}}$ & $100 \pm 0.00^{\mathrm{a}}$ & $95.76 \pm 0.18^{\mathrm{c}}$ \\
\hline 5 & $100 \pm 0.00^{\mathrm{a}}$ & $96.20 \pm 0.13^{\mathrm{a}}$ & $100 \pm 0.00^{\mathrm{a}}$ & $100 \pm 0.00^{\mathrm{a}}$ \\
\hline
\end{tabular}

Table 6. Phytotoxic effect of $\beta$-eudesmol and glyphosate on seedling growth of A. retroflexus. ${ }^{\mathrm{a}} \pm$ Standard error $(n=30)$. ${ }^{b}$ Different letters represent a significant difference at $p<0.05$ level according to Fisher's LSD test.

\begin{tabular}{|l|l|l|l|l|}
\hline \multirow{2}{*}{ Concentration $(\mathbf{m g} / \mathbf{m L})$} & \multicolumn{2}{l|}{ Root inhibition (\%) } & \multicolumn{2}{l|}{ Shoot inhibition (\%) } \\
\cline { 2 - 5 } & $\boldsymbol{\beta}$-Eudesmol & Glyphosate & $\beta$-Eudesmol & Glyphosate \\
\hline 0.25 & $63.34 \pm 7.5^{\mathrm{a}}$ & $79.08 \pm 0.10^{\mathrm{c}}$ & $68.96 \pm 6.78^{\mathrm{a}}$ & $73.60 \pm 0.06^{\mathrm{c}}$ \\
\hline 0.5 & $100 \pm 0.00^{\mathrm{a}}$ & $93.44 \pm 0.23^{\mathrm{b}}$ & $100 \pm 0.00^{\mathrm{a}}$ & $92.80 \pm 0.24^{\mathrm{b}}$ \\
\hline 1 & $100 \pm 0.00^{\mathrm{a}}$ & $100 \pm 0.00^{\mathrm{a}}$ & $100 \pm 0.00^{\mathrm{a}}$ & $100 \pm 0.00^{\mathrm{a}}$ \\
\hline 2.5 & $100 \pm 0.00^{\mathrm{a}}$ & $100 \pm 0.00^{\mathrm{a}}$ & $100 \pm 0.00^{\mathrm{a}}$ & $100 \pm 0.00^{\mathrm{a}}$ \\
\hline 5 & $100 \pm 0.00^{\mathrm{a}}$ & $100 \pm 0.00^{\mathrm{a}}$ & $100 \pm 0.00^{\mathrm{a}}$ & $100 \pm 0.00^{\mathrm{a}}$ \\
\hline
\end{tabular}

Table 7. Phytotoxic effect of $\beta$-eudesmol and glyphosate on seedling growth of $P$. annua. ${ }^{\mathrm{a}} \pm$ Standard error $(n=30) .{ }^{\mathrm{b}}$ Different letters represent a significant difference at $p<0.05$ level according to Fisher's LSD test.

and on P. апnua was 8.29 and 101.88 times of leaf and stem oils, respectively. Further experiment revealed that $\beta$-eudesmol (10.10\%), which was a dominant compound in flower oil but not detected in leaf and stem oils, was most likely responsible for the potent phytotoxic activity of flower oil by comparing the strength of $\beta$-eudesmol and flower oil. $\beta$-eudesmol was found to possess antiangiogenic, antimutagenic, anticancer, antioxidant activity and so on, however there is no report on its phytotoxicity ${ }^{21,31-36}$.

$\beta$-Eudesmol has been previously reported to be abundant in Drimys winteri oil (7.27\%), and in the drupe essential oil of Citharexylum spinosum as the most abundant component (33.1\%) and other species; in some cases, essential oils containing $\beta$-eudesmol were reported to exert phytotoxic activity, such as Geranium wilfordii root oil (4.18\% $\beta$-eudesmol), Helichrysum italicum essential oil (1.4\% $\beta$-eudesmol), Achillea wilhelmsii essential oil ( $2.7 \%$ $\beta$-eudesmol), and Rhynchosia minima ( $0.79 \% \beta$-eudesmol and $16.38 \%$-eudesmol, an analog of $\beta$-eudesmol), however it is difficult to ascribe the activity to certain constituent (s), especially considering the fact that in most 
cases $\beta$-eudesmol was not even the major components in the oils with phytotoxic activity. It is noteworthy to mention that the current report is the first on the phytotoxicity of $\beta$-eudesmol ${ }^{37-39}$.

Essential oils produced by various plant species are found to possess a number of biological activities including phytotoxic activity. As a matter of fact, not only essential oils but also their constituent such as thymol, $\beta$-myrcene, limonene, camphor, eucalyptol, geraniol, $\alpha$ - and $\beta$-pinene, borneol, etc. have been reported to play roles as plant growth regulatory agents ${ }^{40-42}$.

Besides, there are successful examples of utilizing essential oil or oil constituents as commercial herbicides. For example, the herbicide cinmethylin is the derivative of 1,4-cineole, which is a natural phytotoxin that can be found in the volatile oils of many plant species; clove oil is used as the major component in the commercial herbicide Burnout II (Bonide Products Inc., New York) ${ }^{43,44}$. The potent inhibitory activity of the flower oil and its major component, $\beta$-eudesmol, indicated their potential value of being further explored as environment friendly herbicide; meanwhile, the fact that the aboveground plant parts of $O$. acanthium can release volatile compounds into the surroundings support the hypothesis proposed by Watanabe that allelopathy might contribute to the widespread of this species ${ }^{45,46}$.

\section{Methods}

Plant materials. Aboveground plant parts of O. acanthium were harvested in Changji city, Xinjiang province, China, in July 2018 (N43 ${ }^{\circ} 98^{\prime} 53^{\prime \prime}$, E86 $\left.46^{\prime} 22^{\prime \prime}\right)$. Specimens were authenticated and identified by Dr. Wenjun $\mathrm{Li}$, and a voucher specimen (XJBI018326) was deposited at the herbarium of Xinjiang Institute of Ecology and Geography, Chinese Academy of Sciences. Plant materials were separated into flowers, leaves, and stems for further process.

Essential oil extraction. Essential oils were obtained by performing traditional boiling hydrodistillation procedure. Fresh flowers, leaves, and stems of O. acanthium ( $200 \mathrm{~g}$ were used for each plant organ) were laid out in the flask containing water and the unit is carried to boiling for $4 \mathrm{~h}$. The mixture of water-oil was produced in the flask then condensed in the condenser, where the oils were harvested. The same procedure was repeated until enough oils were obtained for GC/MS analysis and the phytotoxic activity assay. The essential oils were dried over anhydrous $\mathrm{Na}_{2} \mathrm{SO}_{4}$ and stored at $4{ }^{\circ} \mathrm{C}$ for the following $\mathrm{GC} / \mathrm{MS}$ analysis and bioassay experiments.

GC/MS analysis. GC/MS was performed to determine the chemical constituents of $O$. acanthium oil using a 7890A/5975C GC-MS system with an automatic injector (Agilent Technologies, Palo Alto, CA, USA) equipped with FID and a DB-5MS 5\% Phenyl Methyl Silox column $(30 \mathrm{~m} \times 0.25 \mathrm{~mm}$; film thickness $0.25 \mu \mathrm{m})$. The experimental conditions were programmed as follows: Helium (carrier gas) at a flow rate of $1 \mathrm{~mL} / \mathrm{min}$; the temperature of the oven was first maintained at $60^{\circ} \mathrm{C}$ for $5 \mathrm{~min}$, then programmed from 60 to $280^{\circ} \mathrm{C}$ at a rate of $3^{\circ} \mathrm{C} / \mathrm{min}$; injector and detector temperature: $280^{\circ} \mathrm{C}$; sample injection volume: $0.1 \mu \mathrm{L}$ (the sample was first diluted in hexane at high-performance liquid chromatography grade at 1:100, v/v); split ratio: 50:1; mass spectra were taken at $70 \mathrm{eV}$ with mass range from $\mathrm{m} / \mathrm{z} 40-800 \mathrm{amu}$. Identification of essential oil constituents was achieved based on comparison of their mass spectra and retention indices (RI, calculated by linear interpolation relative to retention times of a standard mixture of C7-C40 n-alkanes at the same chromatographic conditions s) with the National Institute of Standards and Technology (NIST 14) library database and a home-made library obtained from genuine compounds, as well as those given in the published literature ${ }^{11,25}$. Relative amounts of individual constituents were calculated based on GC peak areas without using correction factors.

Phytotoxic bioassay. Strength of phytotoxicity of $O$. acanthium essential oils obtained from different plant parts along with the oil's major components were investigated by performing bioassays against Amaranthus retroflexus L. (dicot) and Poa annua L. (monocot) using glyphosate (Roundup, Monsanto Co., St. Louis, MO, USA) being the positive control; these 2 species were chosen because they can be found growing alongside $O$. acanthium in the same habitat. The essential oils' major constituents including tricosane, tetracosane, pentacosane, hexacosane, heptacosane, octacosane, nonacosane, and $\beta$-eudesmol were purchased from Alfa Aesar (Shanghai, China); purity of all compounds were $98 \%$. Seeds of receiver species were surface sterilized with $2 \%$ sodium hypochlorite before use. The oils and the major components were first dissolved in DMSO $(0.1 \%$ final concentration) and then diluted with distilled water containing Tween 80 (final concentration $0.02 \%$ ) to give solutions at $0.25,0.5,1,2.5$, and $5 \mathrm{mg} / \mathrm{mL}$ for the assay (a preliminary experiment was conducted and results demonstrated that DMSO and Tween 80 at such concentrations did not affect seedling growth of $A$. retroflexus and $P$. annua significantly). Each petri dish $(\varphi 5 \mathrm{~cm})$ received $2 \mathrm{~mL}$ solutions (controls received $2 \mathrm{~mL}$ of distilled $\mathrm{H}_{2} \mathrm{O}$ containing $0.1 \%$ DMSO and $0.02 \%$ Tween 80 ) and 10 sterilized seeds of receiver species. Petri dishes were sealed with parafilm and kept in a growth cabinet at $25^{\circ} \mathrm{C}$ with a 12 -h photoperiod. A. retroflexus and P. annua seedlings were measured after 5 days and 7 days of incubation, respectively, due to relative slow development of $P$. annua seedlings. Three replicates were performed for the assays $(n=30)^{46}$.

Statistical analyses. ANOVA $(p<0.05)$ was applied to determine the significance of phytotoxicity triggered by the $O$. acanthium essential oils and the major components, followed by analysis using Fisher's LSD test $(p<0.05)$. Experimental results were statistically analyzed using SPSS 13.0 software package and presented as means of 3 replicates \pm standard error. 


\section{Conclusion}

The current study is the first report on the chemical profile and phytotoxicity of O. acanthium essential oil, and on the phytotoxicity of $\beta$-eudesmol, which is speculated to be the responsible compound of flower oil, indicating their potential of being further explored as bioherbicides; flower oil and stem oil were found to be rich in $n$-alkanes, implying that $O$. acanthium has the potential to be further explored as a natural source to produce alkanes for industrial purpose.

Received: 14 May 2020; Accepted: 30 July 2020

Published online: 11 August 2020

\section{References}

1. Rother, C. Administration of Cardiodoron" in patients with functional cardiovascular disorders and/or sleep disorder-results of a prospective, non-interventional study. Forsch. Komplementmed. 20, 334-344 (2013).

2. Cavers, P. B., Qaderi, M. M., Threadgill, P. F. \& Steel, M. G. The biology of Canadian weeds. 147. Onopordum acanthium L. Can. J. Plant Sci. 91, 739-758 (2011).

3. An et al. Flora of Xinjiangensis. Volume 5: Asteraceae. 5, 334-336 (1999).

4. Mucina, L. Syntaxonomy of the Onopordum acanthium communities in temperate and continental Europe. Vegetatio 81, 107-115 (1989).

5. Zahed, N., Hosni, K., Brahim, N. B., Kallel, M. \& Sebei, H. Allelopathic effect of Schinus molle essential oils on wheat germination. Acta Physiol. Plant. 32, 1221-1227 (2010).

6. Abusamra, Y. A. K. et al. Evaluation of putative cytotoxic activity of crude extracts from Onopordum acanthium leaves and Spartium junceum flowers against the U-373 glioblastoma cell line. Pak. J. Pharm. Sci. 28, 1225-1232 (2015).

7. Csupor-Löfller, B., Zupkó, I., Molnár, J., Forgo, P. \& Hohmann, J. Bioactivity-guided isolation of antiproliferative compounds from the roots of Onopordum acanthium. Nat. Prod. Commun. 9, 337-340 (2014).

8. Daci, A., Gold-Binder, M., Garzon, D., Patea, A. \& Beretta, G. Standardization of solvent extracts from Onopordum acanthium fruits by GC-MS, HPLC-UV/DAD, HPLC-TQMS and ${ }^{1} \mathrm{H}-\mathrm{NMR}$ and evaluation of their inhibitory effects on the expression of IL-8 and e-selectin in immortalized endothelial cells (HUVECtert). Nat. Prod. Commun. 9, 945-948 (2014).

9. Lajter, I. et al. Anti-inflammatory activity of Onopordum acanthium extracts and isolated compounds. Planta Med. 80, 255 (2014).

10. Réthy, B. et al. Antiproliferative activity of Hungarian Asteraceae species against human cancer cell lines. Part I. Phytother. Res. 21, $1200-1208$ (2007)

11. Shao, H. et al. Chemical composition and phytotoxic activity of Seriphidium terrae-albae (Krasch.) Poljakov (Compositae) essential oil. Chem. Biodivers. 15, e1800348 (2018).

12. Tsuneki, H. et al. Antiangiogenic activity of $\beta$-eudesmol in vitro and in vivo. Eur. J. Pharmacol. 512, 105-115 (2005).

13. Garsiya, E. R., Konovalov, D. A., Shamilov, A. A., Glushko, M. P. \& Orynbasarova, K. K. Traditional medicine plant, Onopordum acanthium L. (Asteraceae): chemical composition and pharmacological research. Plants. 8(2), 40 (2019).

14. Wei, C. et al. Chemical composition and allelopathic, phytotoxic and pesticidal activities of Atriplex cana LEDEB. (Amaranthaceae) essential oil. Chem. Biodivers. https://doi.org/10.1002/cbdv.201800595 (2019).

15. Brutti, C. B., Pardo, M. F., Caffini, N. O. \& Natalucci, C. L. Onopordum acanthium L. (Asteraceae) flowers as coagulating agent for cheesemaking. LWT Food Sci. Technol. 45, 172-179 (2012).

16. Fernald, M. L., Kinsey, A. C. \& Rollins, R. C. Edible Wild Plants of Eastern North America (Courier Corporation, Chelmsford, 1996).

17. Arfaoui, M. O., Renaud, J., Ghazghazi, H., Boukhchina, S. \& Mayer, P. Variation in oil content, fatty acid and phytosterols profile of Onopordum acanthium L. during seed development. Nat. Prod. Res. 28, 2293-2300 (2014).

18. Gerçel, H. F. The effects of different catalysts on the pyrolysis of thistle, Onopordum acanthium L. Energy Sources 35, 791-799 (2013).

19. Matthaus, B., Ozcan, M. M. \& Al-Juhaimi, F. Fatty acid, tocopherol, and mineral contents of Onopordum acanthium seed and oil. Chem. Nat. Compd. 50, 1092-1093 (2014).

20. Verdeguer, M. et al. Herbicidal activity of Peumus boldus and Drimys winterii essential oils from Chile. Molecules 16, 403-411 (2011).

21. El Ayeb-Zakhama, A. et al. Chemical composition and allelopathic potential of essential oils from Citharexylum spinosum L. grown in Tunisia. Chem. Biodivers. https://doi.org/10.1002/cbdv.201600225 (2017).

22. De, J., Lu, Y., Ling, L., Peng, N. \& Zhong, Y. Essential oil composition and bioactivities of Waldheimia glabra (Asteraceae) from Qinghai-Tibet plateau. Molecules 22, 460-466 (2017).

23. Gilani, S. A., Fujii, Y., Sugano, M. \& Watanabe, K. N. Chemotypic variations and phytotoxic studies of essential oils of endemic medicinal plant, Seriphidium kurramense, from Pakistan. J. Med. Plants Res. 4, 309-315 (2010).

24. Grulová, D. et al. Composition and bioactivity of essential oils of Solidago spp. and their impact on radish and garden cress. Allelopathy J39, 129-142 (2016).

25. Kaur, S., Singh, H. P., Mittal, S., Batish, D. R. \& Kohli, R. K. Phytotoxic effects of volatile oil from Artemisia scoparia against weeds and its possible use as a bioherbicide. Ind. Crops Prod. 32, 54-61 (2010).

26. Bouazzi, S. et al. Chemical composition and antioxidant activity of essential oils and hexane extract of Onopordum arenarium from Tunisia. J. Chromatogr. Sci. 58, 287-293 (2020).

27. Polatoğlu, K., Demirci, B. \& Başer, K. H. C. High amounts of n-alkanes in the composition of Asphodelus aestivus Brot. flower essential oil from Cyprus. J. Oleo Sci. 65, 867-870 (2016).

28. Lazari, D. M., Skaltsa, H. D. \& Constantinidis, T. Volatile constituents of Centaurea raphanina Sm. Subsp. mixta (DC.) Runemark and C. spruneri Boiss. \& Heldr. (Asteraceae), growing wild in Greece. Flavour. Frag. J. 14, 415-418 (1999).

29. Klocke, J. A., Balandrin, M. F., Adams, R. P. \& Kingsford, E. Insecticidal chromenes from the volatile oil of Hemizonia fitchii. J. Chem. Ecol. 11, 701-712 (1985).

30. Heinrich, G., Pfeifhofer, H. W., Stabentheiner, E. \& Sawidis, T. Glandular hairs of Sigesbeckia jorullensis Kunth (Asteraceae): morphology, histochemistry and composition of essential oil. Ann. Bot. 89, 459-469 (2002).

31. El Ayeb-Zakhama, A. et al. Chemical composition and allelopathic potential of essential oils from Tipuana tipu (Benth.) Kuntze cultivated in Tunisia. Chem. Biodivers. 13, 309-318 (2016).

32. Guleria, S. et al. Chemical composition, antioxidant activity and inhibitory effects of essential oil of Eucalyptus teretecornis grown in north-western Himalaya against Alternaria alternata. J. Plant Biochem. Biotechnol. 21, $44-50$ (2012).

33. Miyazawa, M., Shimamura, H., Nakamura, S. I. \& Kameoka, H. Antimutagenic activity of (+)- $\beta$-eudesmol and paeonol from Dioscorea japonica. J. Agric. Food Chem. 44, 1647-1650 (1996). 
34. Plengsuriyakarn, T., Karbwang, J. \& Na-Bangchang, K. Anticancer activity using positron emission tomography-computed tomography and pharmacokinetics of $\beta$-eudesmol in human cholangiocarcinoma xenografted nude mouse model. Clin. Exp. Pharmacol. Physiol. 42, 293-304 (2015).

35. Ul'chenko, N. T., Gusakova, S. D. \& Glishenkova, A. I. Oxygenated triacylglycerols of the lipids of Onopordum acanthium seeds. Chem. Nat. Compd. 29, 578-581 (1993).

36. Watanabe, Y. et al. Phytotoxic potential of Onopordum acanthium L. (Asteraceae). Chem. Biodivers. 11, 1247-1255 (2014).

37. Çakır, A. et al. Phytotoxic and insecticidal properties of essential oils and extracts of four Achillea species. Rec. Nat. Prod. 10, 154-167 (2015).

38. Mancini, E., De Martino, L., Marandino, A., Scognamiglio, M. R. \& De Feo, V. Chemical composition and possible in vitro phytotoxic activity of Helichrsyum italicum (Roth) Don ssp. italicum. Molecules 16, 7725-7735 (2011).

39. Rammohan, A., Reddy, G. M., Bhaskar, B. V., Gunasekar, D. \& Zyryanov, G. V. Phytochemistry and pharmacological activities of the genus Rhynchosia: a comprehensive review. Planta 251, 9 (2020).

40. Martino, L. D., Mancini, E., Almeida, L. F. R. D. \& Feo, V. D. The antigerminative activity of twenty-seven monoterpenes. Molecules 15, 6630-6637 (2010).

41. Zhou, S. et al. Chemical composition, phytotoxic, antimicrobial and insecticidal activity of the essential oils of Dracocephalum integrifolium. Toxins 11, 598 (2019).

42. Ahuja, N., Batish, D. R., Singh, H. P. \& Kohli, R. K. Herbicidal activity of eugenol towards some grassy and broad-leaved weeds. J. Pest Sci. 88(1), 209-218 (2015).

43. Grayson, B. T. et al. The physical and chemical properties of the herbicide cinmethylin (SD 95481). Pestic. Sci. 21, 143-153 (1987).

44. Qaderi, M. M., Cavers, P. B. \& Bernards, M. A. Isolation and structural characterization of a water-soluble germination inhibitor from Scotch thistle (Onopordum acanthium) cypselas. J. Chem. Ecol. 29, 2425-2438 (2003).

45. Young, J. A. \& Evans, R. A. Control and ecological studies of scotch thistle. Weed Sci. 17, 60-63 (1969).

46. Sharifi, N. et al. Isolation, identification and molecular docking studies of a new isolated compound, from Onopordon acanthium: a novel angiotensin converting enzyme (ACE) inhibitor. J. Ethnopharmacol. 148, 934-939 (2013).

\section{Acknowledgements}

This work was supported by the Second Tibetan Plateau Scientific Expedition and Research (STEP) program (2019QZKK0502), the Strategic Priority Research Program of Chinese Academy of Sciences (XDA2006030201), the National Natural Science Foundation of China (31770586), and the Taishan Scholars Program of Shandong, China (ts201712071).

\section{Author contributions}

C.W.: conceptualization, methodology, data curation, writing-original draft preparation. S.Z.: investigation, software, visualization. K.S.: investigation; data curation. C.Z.: investigation, formal analysis. H.S.: conceptualization, supervision, project administration, funding acquisition.

\section{Competing interests}

The authors declare no competing interests.

\section{Additional information}

Correspondence and requests for materials should be addressed to H.S.

Reprints and permissions information is available at www.nature.com/reprints.

Publisher's note Springer Nature remains neutral with regard to jurisdictional claims in published maps and institutional affiliations.

(c) (i) Open Access This article is licensed under a Creative Commons Attribution 4.0 International cc) License, which permits use, sharing, adaptation, distribution and reproduction in any medium or format, as long as you give appropriate credit to the original author(s) and the source, provide a link to the Creative Commons license, and indicate if changes were made. The images or other third party material in this article are included in the article's Creative Commons license, unless indicated otherwise in a credit line to the material. If material is not included in the article's Creative Commons license and your intended use is not permitted by statutory regulation or exceeds the permitted use, you will need to obtain permission directly from the copyright holder. To view a copy of this license, visit http://creativecommons.org/licenses/by/4.0/.

(C) The Author(s) 2020 